\title{
Stability Analysis of Relativistic Electron Beams in a Wiggler with Harmonic Gyro-Resonance Using the Lie Perturbation Method
}

\author{
Kenji IMADERA and Yasuaki KISHIMOTO \\ Department of Fundamental Energy Science, Graduate School of Energy Science, Kyoto University, \\ Gokasho, Uji, Kyoto 611-0011, Japan
}

(Received 8 July 2010 / Accepted 9 November 2010)

\begin{abstract}
The non-canonical Lie perturbation method for analyzing relativistic electron beams in free electron lasers [Y. Kishimoto et al., Phys. Plasmas 2, 1316 (1995)] is extended to the case with harmonic gyro-resonance due to the coexistence of a focusing wiggler and an axial guiding field, which allow the maximum beam current to be increased. By using non-canonical guiding-center variables, we have solved the particle motion not only far from the harmonic gyro-resonance but also near the resonance. Far from the resonance, the maximum beam current is found to increase in proportion to $\left(B_{\mathrm{g}} / B_{\mathrm{w}}\right)^{2}\left(B_{\mathrm{w}}\right.$ and $B_{\mathrm{g}}$ are the strength of the wiggler and guiding fields, respectively). On the other hand, near the resonance, the beam is found to be confined in a finite radial region and then transmitted because of higher order secular perturbations.
\end{abstract}

(c) 2011 The Japan Society of Plasma Science and Nuclear Fusion Research

Keywords: non-canonical variable, Lie transformation, stability analysis, focusing wiggler

DOI: $10.1585 /$ pfr.6.1201004

Stable propagation of beams with high current and low emittance is crucial for obtaining high performance in free electron lasers (FELs). In general, focusing fields are incorporated to prevent beam particles from diverging. A self-focusing wiggler [1], in which the surface of the wiggler magnet consists of parabolically curved pole faces, has been introduced and found to be superior because it leads to stable beam propagation, and maintains the axial phase relationship between the beam particles and radiation field [2].

To further increase the beam current, Sakamoto et al. [3] superposed an axial guiding field on the selffocusing wiggler and demonstrated the advantages of this configuration. Meanwhile, they also observed a deterioration of beam propagation due to the resonance between the wiggler and cyclotron motions. They determined the resonance frequency by using a heuristic averaging method; however, the details of the stability conditions for beam propagation in the presence of a radial electric field and/or resonance have not been studied. The difficulty mainly originates from the complex relativistic particle motion in such plural magnetic fields.

Kishimoto et al. [4] explored a methodology that uses a non-canonical Lie perturbation method based on the phase-space Lagrangian formalism [5] to analyze relativistic electron beams in FELs, and proved that the method is efficient and powerful for studying the effect of higher order perturbations. Specifically, non-canonical variables are found to be superior for simplifying perturbation analysis in the relativistic regime.

author'se-mail: imadera@centeriae.kyoto-u.ac.jp
In this study, we have extended the method to the case with harmonic gyro-resonance. By selecting a coordinate transformation in terms of non-canonical guiding-center variables, we performed a stability analysis where higher order perturbations are properly taken into account.

Here, we start with the non-canonical one-form

$$
\begin{aligned}
\gamma_{\mu} \mathrm{d} z^{\mu}= & \left(p_{x}+\frac{e}{c} A_{x}\right) \mathrm{d} x+\left(p_{y}+\frac{e}{c} A_{y}\right) \mathrm{d} y \\
& +\left(p_{t}-e \phi\right) \mathrm{d} t-K \mathrm{~d} z
\end{aligned}
$$

where $\left(p_{x}, p_{y}\right)$ correspond to the mechanical transverse momentums, and $p_{t}=-\gamma m c^{2}$ is the beam energy [4]. $K$ is the new Hamiltonian, given by

$$
K=-\sqrt{\alpha^{2}-p_{x}^{2}-p_{y}^{2}}-\frac{e}{c} A_{z}
$$

where $\alpha=\sqrt{\gamma^{2}-1} m c$ represents the total momentum. Note that in Eq. (1), the axial coordinate $z$ is selected as the independent variable instead of time $t$. In addition, the vector potential $\boldsymbol{A}$ is not explicitly included in the root in Eq. (2), which is an important characteristic in the following perturbation analysis.

A focusing wiggler with an axial guiding field is given by

$$
\begin{aligned}
& B_{x}=B_{\mathrm{w}} \sinh \left(k_{x} x\right) \sinh \left(k_{y} y\right) \cos \left(k_{\mathrm{w}} z\right) \\
& B_{y}=B_{\mathrm{w}} \cosh \left(k_{x} x\right) \cosh \left(k_{y} y\right) \cos \left(k_{\mathrm{w}} z\right), \\
& B_{\mathrm{z}}=-\sqrt{2} B_{\mathrm{w}} \cosh \left(k_{x} x\right) \sinh \left(k_{y} y\right) \sin \left(k_{\mathrm{w}} z\right)+B_{\mathrm{g}},
\end{aligned}
$$

where $k_{x}=k_{y}=k_{\mathrm{w}} / \sqrt{2}$. The primary wiggler motion is in the $x$-direction and the beam propagates in the $z$-direction. 
The axial guiding field $B_{\mathrm{g}} z$ is assumed to be spatially uniform.

Here, we introduce two smallness parameters that characterize beam dynamics in a wiggler. One is $\varepsilon \sim$ $r_{\mathrm{b}} / \lambda_{\mathrm{w}} \sim r_{\mathrm{b}} / \lambda_{\mathrm{g}}\left(r_{\mathrm{b}}\right.$ : typical beam radius, $\lambda_{\mathrm{w}} \equiv 2 \pi / k_{\mathrm{w}}$ : wiggler pitch, $\lambda_{\mathrm{g}} \equiv 2 \pi / k_{\mathrm{g}}=2 \pi c \alpha / e B_{\mathrm{g}}$ : effective gyro radius); the radial deviation of electrons from the design orbit, i.e., $\sqrt{x^{2}+y^{2}}$, is assumed to be of the same order as $r_{\mathrm{b}}$. The other is $\delta \sim p_{\mathrm{w}} / \alpha$, where $p_{\mathrm{w}}=\sqrt{2} \hat{K} m c$ ( $\hat{K}=e B_{\mathrm{w}} / \sqrt{2} m c^{2} k_{\mathrm{w}}$ : K-parameter) is the transverse momentum of electrons due to the wiggler motion. The relationship between the above two smallness parameters is based on the experimental conditions. Here, we consider the simple case of $\delta \sim \varepsilon$.

To solve the particle motion in such a configuration with multiple magnetic fields, we have a choice of coordinate transformations, which depend on whether the harmonic gyro-resonance condition given by $k_{\mathrm{w}} \sim \pm n k_{\mathrm{g}}$ is satisfied. Assuming that the configuration is sufficiently far from such condition, we perform non-canonical transformation to the lowest-order wiggler-center coordinate

$z^{\mu}=\left(z ; x, y, t, p_{x}, p_{y}, p_{t}\right) \mapsto Z^{\mu}=\left(z ; X, Y, t, U, V, p_{t}\right)$.

Here, $(X, Y, U, V)$ are given by

$$
\begin{aligned}
& X=x-C_{\mathrm{w}} \frac{p_{\mathrm{w}}}{\alpha k_{\mathrm{w}}} \cos k_{\mathrm{w}} z, \\
& Y=y+C_{\mathrm{g}} \frac{p_{\mathrm{w}}}{\alpha k_{\mathrm{w}}} \sin k_{\mathrm{w}} z, \\
& U=p_{x}+C_{\mathrm{w}} p_{\mathrm{w}} \sin k_{\mathrm{w}} z, \\
& V=p_{y}+C_{\mathrm{g}} p_{\mathrm{w}} \cos k_{\mathrm{w}} z,
\end{aligned}
$$

where $C_{\mathrm{w}}=k_{\mathrm{w}}^{2} /\left(k_{\mathrm{w}}^{2}-k_{\mathrm{g}}^{2}\right)$ and $C_{\mathrm{g}}=k_{\mathrm{w}} k_{\mathrm{g}} /\left(k_{\mathrm{w}}^{2}-k_{\mathrm{g}}^{2}\right)$. These new coordinates are determined from the lowest order solutions on the vertical plane for which only the primary wiggler and guiding fields are taken into account. The corresponding covariant vector $\Gamma_{\mu} \mathrm{d} Z^{\mu}$ is determined from the relationship $\Gamma_{\mu} \mathrm{d} Z^{\mu}=\gamma_{v}\left(\partial z^{\mu} / \partial Z^{v}\right) \mathrm{d} Z^{\mu}$ and then simplified by retaining only the secular perturbations through the Lie perturbation method. We obtain a new one-form up to the order of $O\left(\varepsilon^{4}\right)$, in which each order is given by

$$
\begin{aligned}
& \Gamma_{\mu}^{(0)} \mathrm{d} Z^{\mu}=p_{t} \mathrm{~d} t+\alpha \mathrm{d} z \\
& \Gamma_{\mu}^{(2)} \mathrm{d} Z^{\mu}=\left(U-\alpha k_{\mathrm{g}} Y\right) \mathrm{d} X+V \mathrm{~d} Y-C_{\mathrm{w}}^{2} \frac{p_{\mathrm{w}}^{2}}{4 \alpha} \mathrm{d} z \\
& \Gamma_{\mu}^{(4)} \mathrm{d} Z^{\mu}=\left\{-\frac{U^{2}+V^{2}}{2 \alpha}-C_{\mathrm{w}} \frac{p_{\mathrm{w}}^{2}}{8 \alpha} k_{\mathrm{w}}^{2}\left(X^{2}+Y^{2}\right)\right. \\
& \left.\quad+\frac{G e I_{\mathrm{b}}}{c^{2}\left(k_{\mathrm{w}} r_{\mathrm{b}}\right)^{2}} k_{\mathrm{w}}^{2}\left(X^{2}+Y^{2}\right)+C_{\mathrm{w}}^{3} \frac{p_{\mathrm{w}}^{4}}{32 \alpha^{3}}-C_{\mathrm{g}}^{2} \frac{p_{\mathrm{w}}^{2}}{4 \alpha}\right\} \mathrm{d} z
\end{aligned}
$$

where $\left.G=(m c / \alpha)^{2}\left[1+C_{\mathrm{w}}^{2}\left(k_{\mathrm{w}}^{2}+k_{\mathrm{g}}^{2}\right) \hat{K} / 2 k_{\mathrm{w}}^{2}\right)\right]$ and $\Gamma_{\mu}^{(1)} \mathrm{d} Z^{\mu}=\Gamma_{\mu}^{(3)} \mathrm{d} Z^{\mu}=0$. Here, the radial electric field and pinching force are taken into account by assuming that the beam distribution is radially uniform with current $I_{\mathrm{b}}$ [4]. The order of the current effect given by the third term on the right-hand side (RHS) of Eq. (8) depends on the beam parameters and is not specified so far. Here, the order of the self-focusing effect is $O\left(\varepsilon^{4}\right)$, which should balance the current effect in the limit of maximum beam current, so we consider the order of the current effect to be also $O\left(\varepsilon^{4}\right)$.

Then, the equations of motion are derived from Eqs. (6)-(8) as

$$
\begin{aligned}
& \frac{\mathrm{d} X}{\mathrm{~d} z}=\frac{U}{\alpha}, \\
& \frac{\mathrm{d} Y}{\mathrm{~d} z}=\frac{V}{\alpha}, \\
& \frac{\mathrm{d} U}{\mathrm{~d} z}=-\left[C_{\mathrm{w}} \frac{p_{\mathrm{w}}^{2}}{4 \alpha}-\frac{2 G e I_{\mathrm{b}}}{c^{2}\left(k_{\mathrm{w}} r_{\mathrm{b}}\right)^{2}}\right] k_{\mathrm{w}}^{2} X+k_{\mathrm{g}} V, \\
& \frac{\mathrm{d} V}{\mathrm{~d} z}=-\left[C_{\mathrm{w}} \frac{p_{\mathrm{w}}^{2}}{4 \alpha}-\frac{2 G e I_{\mathrm{b}}}{c^{2}\left(k_{\mathrm{w}} r_{\mathrm{b}}\right)^{2}}\right] k_{\mathrm{w}}^{2} Y-k_{\mathrm{g}} U .
\end{aligned}
$$

Equations (9)-(12) describe a long-period helical motion in which the wave number is given by

$$
k_{\mathrm{h}}= \pm \sqrt{\frac{2 k_{\beta}^{2}+k_{\mathrm{g}}^{2} \pm \sqrt{4 k_{\beta}^{2} k_{\mathrm{g}}^{2}+k_{\mathrm{g}}^{4}}}{2}}
$$

where

$$
k_{\beta}=\sqrt{C_{\mathrm{w}} \frac{p_{\mathrm{w}}^{2}}{4 \alpha^{2}}-\frac{2 G e I_{\mathrm{b}}}{c^{2}\left(k_{\mathrm{w}} r_{\mathrm{b}}\right)^{2} \alpha}} k_{\mathrm{w}} .
$$

From Eqs. (13) and (14), the maximum beam current, above which beam propagation is not expected, is estimated as

$$
I_{\mathrm{b}, \max }=\frac{c^{2}\left(k_{\mathrm{w}} r_{\mathrm{b}}\right)^{2} p_{\mathrm{w}}^{2}}{8 G e \alpha}\left[C_{\mathrm{w}}+\frac{1}{2}\left(\frac{B_{\mathrm{g}}}{B_{\mathrm{w}}}\right)^{2}\right]
$$

From Eq. (15), the maximum beam current is found to increase in proportion to $\left(B_{\mathrm{g}} / B_{\mathrm{W}}\right)^{2}$ because of the axial guiding field.

To solve the particle motion near the harmonic resonance between the wiggler and cyclotron motions given by $k_{\mathrm{w}} \sim \pm n k_{\mathrm{g}}$, we need to retain not only the secular terms but also the resonance ones, which correspond to longperiod perturbations. The guiding-center coordinate is appropriate to distinguish such terms, so we change the noncanonical transformation to

$z^{\mu}=\left(z ; x, y, t, p_{x}, p_{y}, p_{t}\right) \mapsto Z^{\mu}=\left(z ; X, Y, t, \mu, \theta, p_{t}\right)$

Here, $(X, Y)$ and $(\mu, \theta)$ represent the guiding-center vari- 
ables for gyro motion, which are given by

$$
\begin{aligned}
& X=x-C_{\mathrm{w}} \frac{p_{\mathrm{w}}}{\alpha k_{\mathrm{w}}} \cos k_{\mathrm{w}} z-\sqrt{\frac{2 \mu}{\alpha k_{\mathrm{g}}}} \cos \left(k_{\mathrm{g}} z+\theta\right), \\
& Y=y+C_{\mathrm{g}} \frac{p_{\mathrm{w}}}{\alpha k_{\mathrm{w}}} \sin k_{\mathrm{w}} z+\sqrt{\frac{2 \mu}{\alpha k_{\mathrm{g}}}} \sin \left(k_{\mathrm{g}} z+\theta\right), \\
& \mu=\frac{\left(p_{x}+C_{\mathrm{w}} p_{\mathrm{w}} \sin k_{\mathrm{w}} z\right)^{2}+\left(p_{y}+C_{\mathrm{g}} p_{\mathrm{w}} \cos k_{\mathrm{w}} z\right)^{2}}{2 \alpha k_{\mathrm{g}}}, \\
& \theta=\tan ^{-1}\left(\frac{p_{x}+C_{\mathrm{w}} p_{\mathrm{w}} \sin k_{\mathrm{w}} z}{p_{y}+C_{\mathrm{g}} p_{\mathrm{w}} \cos k_{\mathrm{w}} z}\right)-k_{\mathrm{g}} z .
\end{aligned}
$$

Note that $\theta$ represents the phase shift of gyration motion, which is slowly changed by higher order perturbations. Performing similar calculations as those for Eqs. (6)-(8), we obtain a simplified new one-form, also up to $O\left(\varepsilon^{4}\right)$

$\Gamma_{\mu}^{(0)} \mathrm{d} Z^{\mu}=p_{t} \mathrm{~d} t+\alpha \mathrm{d} z$

$\Gamma_{\mu}^{(1)} \mathrm{d} Z^{\mu}=-\alpha k_{\mathrm{g}} Y \mathrm{~d} X$,

$\Gamma_{\mu}^{(2)} \mathrm{d} Z^{\mu}=\mu \mathrm{d} \theta-\left[k_{\mathrm{g}} \mu+\left(C_{\mathrm{w}}^{2}+C_{\mathrm{g}}^{2}\right) \frac{p_{\mathrm{w}}^{2}}{4 \alpha}\right] \mathrm{d} z$,

$\Gamma_{\mu}^{(4)} \mathrm{d} Z^{\mu}=-\left\{\frac{p_{\mathrm{w}}^{4}}{64 \alpha^{3}}\left(3 C_{\mathrm{w}}^{4}-2 C_{\mathrm{w}}^{3}+C_{\mathrm{w}}^{2} C_{\mathrm{g}}^{2}+10 C_{\mathrm{w}} C_{\mathrm{g}}^{2}\right.\right.$

$\left.+3 C_{\mathrm{g}}^{4}\right)+\frac{k_{\mathrm{g}}^{2} \mu^{2}}{2 \alpha}+C_{\mathrm{w}} \frac{p_{\mathrm{w}}^{2}}{8 \alpha} k_{\mathrm{w}}^{2}\left(X^{2}+Y^{2}\right)+\frac{p_{\mathrm{w}} k_{\mathrm{w}}^{2} \mu}{4 \alpha k_{\mathrm{g}}}$

$$
\left.\cdot\left\{\cos \left[\left(k_{\mathrm{w}}+2 k_{\mathrm{g}}\right) z+2 \theta\right]+\sin \left[\left(k_{\mathrm{w}}+2 k_{\mathrm{g}}\right) z+2 \theta\right]\right\}\right\} \mathrm{d} z,
$$

where $\Gamma_{\mu}^{(3)} \mathrm{d} Z^{\mu}=0$. Here, we consider only the case of the second harmonic gyro-resonance given by $k_{\mathrm{w}} \sim-2 k_{\mathrm{g}}$. Note that the fourth term on the RHS of Eq. (21) corresponds to the long-period perturbation. The radial electric field is neglected for simplicity in the following calculation. Then, the new one-form given by Eqs. (18)-(21) yields the equations of motion as

$$
\begin{aligned}
& \frac{\mathrm{d} X}{\mathrm{~d} z}=-C_{\mathrm{w}} \frac{p_{\mathrm{w}}^{2}}{4 \alpha^{2}} \frac{k_{\mathrm{w}}^{2}}{k_{\mathrm{g}}} Y, \\
& \frac{\mathrm{d} Y}{\mathrm{~d} z}=C_{\mathrm{w}} \frac{p_{\mathrm{w}}^{2}}{4 \alpha^{2}} \frac{k_{\mathrm{w}}^{2}}{k_{\mathrm{g}}} X, \\
& \frac{\mathrm{d} \hat{\theta}}{\mathrm{d} z}=k_{\mathrm{w}}+2 k_{\mathrm{g}}+\frac{2 k_{\mathrm{g}}^{2} \mu}{\alpha}+\frac{p_{\mathrm{w}}}{\sqrt{2} \alpha} \frac{k_{\mathrm{w}}^{2}}{k_{\mathrm{g}}} \cos \hat{\theta}, \\
& \frac{\mathrm{d} \mu}{\mathrm{d} z}=\frac{p_{\mathrm{w}}}{\sqrt{2} \alpha} \frac{k_{\mathrm{w}}^{2} \mu}{k_{\mathrm{g}}} \sin \hat{\theta},
\end{aligned}
$$

where $\hat{\theta}=\left(k_{\mathrm{w}}+2 k_{\mathrm{g}}\right) z+2 \theta$. Equations (22) and (23) describe helical motion with a constant radius. Meanwhile, Eqs. (24) and (25) lead to the relationship

$$
\frac{\mathrm{d} \mu}{\mathrm{d} \hat{\theta}}=\frac{\frac{p_{\mathrm{w}}}{\sqrt{2} \alpha} \frac{k_{\mathrm{w}}^{2} \mu}{k_{\mathrm{g}}} \sin \hat{\theta}}{k_{\mathrm{w}}+2 k_{\mathrm{g}}+\frac{2 k_{\mathrm{g}}^{2} \mu}{\alpha}+\frac{p_{\mathrm{w}}}{\sqrt{2} \alpha} \frac{k_{\mathrm{w}}^{2}}{k_{\mathrm{g}}} \cos \hat{\theta}} .
$$

Here, the fourth term in the denominator and also the numerator of Eq. (26) originate from the long-period perturbation, whereas the third term in the denominator arises from the secular perturbation. Note that these three terms are derived from $\Gamma_{\mu}^{(4)} \mathrm{d} Z^{\mu}$, which is neglected in the heuristic averaging method. However, the resonance conditions are qualitatively changed by such higher order terms. Eq. (26) can be solved as

$$
\mu=\frac{\alpha}{2 k_{\mathrm{g}}^{2}}\left\{f(\hat{\theta}) \pm \sqrt{C+\left[\frac{\alpha}{2 k_{\mathrm{g}}^{2}} f(\hat{\theta})\right]^{2}}\right\}
$$

where $f(\hat{\theta})=\left(-k_{\mathrm{w}}-2 k_{\mathrm{g}}-p_{\mathrm{w}} k_{\mathrm{w}}^{2} \cos \hat{\theta}\right) /\left(\sqrt{2} \alpha k_{\mathrm{g}}\right)$ and $C$ is a constant. On the other hand, when the third term in the denominator of Eq. (26) is neglected, which corresponds to the case with small transverse momentum, Eq. (26) yields an approximate solution

$$
\mu=\frac{C}{k_{\mathrm{w}}+2 k_{\mathrm{g}}+\frac{p_{\mathrm{w}}}{\sqrt{2} \alpha} \frac{k_{\mathrm{w}}^{2}}{k_{\mathrm{g}}} \cos \hat{\theta}} .
$$

From Eq. (28), the unstable condition is estimated as

$$
\left|k_{\mathrm{w}}+2 k_{\mathrm{g}}\right|<\frac{p_{\mathrm{w}}}{\sqrt{2} \alpha} \frac{k_{\mathrm{w}}}{k_{\mathrm{g}}} k_{\mathrm{w}}
$$

which gives us a criterion for resonance. Since $\delta \sim$ $p_{\mathrm{w}} / \alpha$ is sufficiently small, the width of the unstable region near the second harmonic gyro-resonance given by $\left(\sqrt{2} p_{\mathrm{w}} / \alpha\right)\left(k_{\mathrm{w}} / k_{\mathrm{g}}\right) k_{\mathrm{w}}$ is sufficiently narrower than the distance between the two adjacent resonances. As a result, the resonances do not overlap in the present case, and the electron motion becomes regular or weakly stochastic.

In conclusion, we performed a systematic stability analysis in a wiggler with harmonic gyro-resonance using the non-canonical Lie perturbation method. Far from the resonance, the maximum beam current is found to increase with $\left(B_{\mathrm{g}} / B_{\mathrm{w}}\right)^{2}$, whereas, near the resonance, the beam is still confined in a finite radial region because of higher order secular perturbations. The methodology of the present stability analysis can be applied to other resonance problems such as cyclotron resonance heating in fusion plasmas.

This work was supported by a Grant-in-Aid from JSPS (No.21340171).

[1] E.T. Scharlemann, J. Appl. Phys. 58, 2154 (1985).

[2] Y. Kishimoto, J. Phys. Soc. Jpn. 65, 3877 (1996).

[3] K. Sakamoto, T. Kobayashi, Y. Kishimoto, S. Kawasaki, S. Musyoki, A. Watanabe, M. Takahashi, H. Ishizuka and M. Shiho, Phys. Rev. Lett. 70, 441 (1993).

[4] Y. Kishimoto, S. Tokuda and K. Sakamoto, Phys. Plasmas 2, 1316 (1995).

[5] J.R. Cary and R.G. Littlejohn, Ann. Phys. 151, 1 (1983). 